\title{
Arc Recognition on Irregular Isothetic Grids and Its Application to Reconstruction of Noisy Digital Contours
}

\author{
Jean-Luc Toutant ${ }^{1,2}$, Antoine Vacavant ${ }^{1,2}$, and Bertrand Kerautret ${ }^{3}$ \\ 1 Clermont Université, Université d'Auvergne, ISIT, BP10448, F-63000 \\ Clermont-Ferrand \\ 2 CNRS, UMR6284, BP10448, F-63000 Clermont-Ferrand \\ \{jean-luc.toutant, antoine.vacavant\}@udamail.fr \\ 3 Université de Nancy, LORIA, UMR7503 CNRS, F-54506, France \\ bertrand.kerautret@univ-lorraine.fr
}

\begin{abstract}
In the present paper, we introduced an arc recognition technique suitable for irregular isothetic object. It is based on the digital inter-pixel (DIP) circle model, a pixel-based representation of the Kovalevsky's circle. The adaptation to irregular image structurations allows us to apply DIP models for circle recognition in noisy digital contours. More precisely, the noise detector from Kerautret and Lachaud (2009) provides a multi-scale representation of the input contour with boxes of various size. We convert them into an irregular isothetic object and, thanks to the DIP model, reduce the recognition of arcs of circles in this object to a simple problem of point separation.
\end{abstract}

Keywords: Arc recognition, Irregular isothetic grid, Digital circle.

\section{Introduction}

The recognition of circles from noisy image data has been widely studied since 60's, and was first introduced by Duda and Hart [5]. The Hough Transform is a powerful tool, robust to noise and to missing parts of an object. Nevertheless, computational and storage requirements of the algorithm are $O\left(n^{3}\right)$ for circles, where $n$ is the size of the input data. Heuristic techniques [15] or nondeterministic algorithms [10] are faster but do not guarantee the same accuracy.

Arcs and circles can also be recognized from extracted contours. P. Damaschke [3] presented a linear-time algorithm by showing that the problem of circle recognition is equivalent to solve a set of inequalities in dimension 3 [11. Online algorithms with a $O\left(n^{4 / 3}\right)$ time complexity are introduced in [116] to segment a digital curve into arcs. All of these techniques are based on the reduction of the problem to a circle separation problem. However, this kind of approaches has the main drawback of not being suitable for noisy contours. In [12], the authors proposed a novel approach, online and linear in time, based on a tangential space. The contour needs to be first polygonized. Thus, noisy

R. Gonzalez-Diaz, M.-J. Jimenez, B. Medrano (Eds.): DGCI 2013, LNCS 7749, pp. 265-276, 2013.

(C) Springer-Verlag Berlin Heidelberg 2013 
contours can be handle by using blurred segments [4 rather than digital segment. In [13, the method is improved with the used of a noise detector [6] to adapt the width of each blurred segment to the data.

In the present paper, we introduce a new digital circle model (DIP-circle) easy to recognize throw circle separation. It extends to irregular isothetic objects and provides arc recognition in such objects. We thus propose a complete unsupervised scheme that aims at recognizing arcs of circle from noisy image data in $O\left(n^{4 / 3}\right)$.

In Section 2, we introduce the definition of circle (DIP-circle) on which we based our work. In Section 3, we give some recalls about irregular isothetic grids and objects. We also present how DIP-circles extend to such grids and allow reduction of the arc recognition problem to a circle separation problem. In Section 4, we present the unsupervised arc recognition scheme and several experiments validating it and giving some clues about its accuracy.

\section{Digital Inter-Pixel Circles}

\subsection{Basic Notions and Recalls}

Let $\left\{\mathbf{e}_{\mathbf{1}}, \mathbf{e}_{\mathbf{2}}\right\}$ denote the canonical basis of the Euclidean vector plane and $\mathbf{o}$ its origin. A point $\mathbf{x}$ is then defined by $\mathbf{x}=x_{1} \mathbf{e}_{\mathbf{1}}+x_{2} \mathbf{e}_{\mathbf{2}}$. Let $\oplus$ be the Minkowski addition such that $\mathcal{A} \oplus \mathcal{B}=\cup_{\mathbf{b} \in \mathcal{B}}\{\mathbf{a}+\mathbf{b}: \mathbf{a} \in \mathcal{A}\}$. It is also known as dilation. The pixel associated to an integer point $\mathbf{x}$ is the dilation $\mathcal{P}(\mathbf{x})=\{\mathbf{x}\} \oplus \mathcal{B}_{\infty}(1 / 2)$ where $\mathcal{B}_{\infty}(1 / 2)$ is the ball of radius $1 / 2$ based on the $\ell^{\infty}$-norm, or, in other words, the square of side 1 centered at $\mathbf{x}$. The interface between two pixels is their intersection. A digital object is a set of integer points or a set of pixels, depending of the context.

Two integer points $\mathbf{x}$ and $\mathbf{y}$ are said to be 4-adjacent if $\|\mathbf{x}-\mathbf{y}\|_{1}=\left|x_{1}-y_{1}\right|+$ $\left|x_{2}-y_{2}\right|=1$. Similarly, two integer points $\mathbf{x}$ and $\mathbf{y}$ are said to be 8 -adjacent if $\|\mathbf{x}-\mathbf{y}\|_{\infty}=\max \left\{\left|x_{1}-y_{1}\right|,\left|x_{2}-y_{2}\right|\right\}=1$. By extension, two pixels are $k$-adjacent $(k=4$ or $k=8)$ if their associated integer points are $k$-adjacent. We also called them $k$-neighbors.

A $k$-path is a sequence of integer points such that every two consecutive points, in the sequence, are $k$-adjacent. If each point appears only one time in the path and admits at most two $k$-neighbors in, then it is called a $k$-arc. A digital object $\mathrm{E}$ is $k$-connected if there exists a $k$-path in E between any two points of $\mathrm{E}$. $\mathrm{A}$ maximum $k$-connected subset of $\mathrm{E}$ is called a $k$-connected component. Let us suppose that the complement of a digital object $\mathrm{E}, \mathbb{Z}^{n} \backslash \mathrm{E}$ admits exactly two $k$ connected components $\mathrm{F}_{1}$ and $\mathrm{F}_{2}$, or, in other words, that there exists no $k$-path joining integer points of $\mathrm{F}_{1}$ and $\mathrm{F}_{2}$, then $\mathrm{E}$ is said to be $k$-separating in $\mathbb{Z}^{n}$.

\subsection{A New Digital Circle Model}

Let $c_{\mathbf{c}, r}$ be the characteristic polynomial of a circle of center $\mathbf{c} \in \mathbb{R}^{2}$ and $r \in \mathbb{R}_{+}$. One has:

$$
\forall \mathbf{x} \in \mathbb{R}^{2}: c_{\mathbf{c}, r}(\mathbf{x})=\left(x_{1}-c_{1}\right)^{2}+\left(x_{2}-c_{2}\right)^{2}-r^{2} .
$$


Definition 1 (Digital Inter-pixel Circle). Let $\mathbf{c} \in \mathbb{R}^{2}$ and $r>\sqrt{2} / 2$. The digital inter-pixel circle (DIP-circle for short), $\mathrm{C}_{\mathrm{DIP}}(\mathbf{c}, r)$ is defined as follows:

$$
\mathrm{C}_{\mathrm{DIP}}(\mathbf{c}, r)=\left\{\begin{array}{ccc} 
& -\left|p_{1}-c_{1}\right|-\left|p_{2}-c_{2}\right|-\frac{1}{2}<c_{\mathbf{c}, r}(\mathbf{p}) \\
\mathbf{p} \in \mathbb{Z}^{2}: & \text { and } \\
& c_{\mathbf{c}, r}(\mathbf{p}) \leq\left|p_{1}-c_{1}\right|+\left|p_{2}-c_{2}\right|-\frac{1}{2}
\end{array}\right\} .
$$

Property 1 (Topology of a DIP-circle). A DIP-circle is a 4-connected and 8separating set.

Let $\mathbf{c} \in \mathbb{R}^{2}$ and $r \in \mathbb{R}$. Let $\mathrm{D}$ be the set of pixels with associated integer point in the disk of center $\mathbf{c}$ and radius $r$. The Kovalevsky's circle [9] of center $\mathbf{c}$ and radius $r$ is then the set of vertices of the inter-pixel boundary of $\mathrm{D}$ (the interfaces of the pixels in $\mathrm{D}$ with pixels not in). The DIP-circle model is strongly related to this type of circles.

Property 2 (DIP-circle and Kovalevsky's circle). The Kovalevsky's circle of center $\mathbf{c}$ and radius $r$ defines the same set of points as $\mathrm{C}_{\mathrm{DIP}}\left(\mathbf{c}^{\prime}, r\right) \oplus(-1 / 2,-1 / 2)$ with $\mathbf{c}^{\prime}=\mathbf{c} \oplus(1 / 2,1 / 2)$.

Kovalevsky's circles and $D I P$-circles are identical up to a translation. Kovalesky's circles thus allow us to link $D I P$-circles with the inter-pixel boundary.

\subsection{DIP-Circles Recognition}

Circle recognition from inter-pixel boundary (vertices and edges) directly reduces to a circle separation problem: inner and outer points are easily obtained with boundary tracking. From DIP-circles - only the vertices of the inter-pixel boundary - the reduction to a circle separation problem is no more trivial. Sometimes $D I P$-circles contain pixels with more than two 4-neighbors and we lack information to track the boundary (one neighbor is the previous pixel in the tracking and one can not decide which of the two remaining pixels is the next one...). Thus, we only consider recognition process of DIP-circle performed on 4 -arcs.

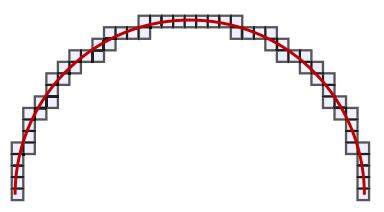

Fig. 1. In a 4-arc, a $D I P$-circle may be recognized by resolving a separation problem between the extremities of each interface between two 4 -adjacent pixels 
Property 3 (Characterization of 4-arcs of DIP-circles). Let $\mathbf{p}$ and $\mathbf{q}$ be two 4-adjacent integer points of $\mathrm{C}_{\mathrm{DIP}}(\mathbf{c}, r)$ such that they form a 4-arc with their 4-neighbors in $\mathrm{C}_{\mathrm{DIP}}(\mathbf{c}, r)$. Then, we have:

- both $\mathcal{P}(\mathbf{p})$ and $\mathcal{P}(\mathbf{q})$ deprived of their interface intersect the circle $\mathcal{C}(\mathbf{c}, r)$,

- the circle $\mathcal{C}(\mathbf{c}, r)$ intersects only in one point the interface between $\mathcal{P}(\mathbf{p})$ and $\mathcal{P}(\mathbf{q})$ deprived of its end points at a distance to $\mathbf{c}$ lower than $r$.

Property 3 allows to easily convert the recognition of arcs of $D I P$-circle into a circle separation problem: the parameters of the recognized $D I P$-circle are the same of those of a circle which separates the both extremities of each interface between two 4-adjacent pixels in a 4 -arc, as in Figure 1 .

\section{$3 \quad$ Digital Inter-Pixel Circles on $\mathbb{I}$-grids}

We first recall the I-grid (Irregular Isothetic grid) model [2 17]:

Definition 2 (2-D $\mathbb{I}$-grid). Let $\mathcal{R}$ be a closed rectangular subset of $\mathbb{R}^{2}$. A 2-D $\mathbb{I}$-grid $G$ is a tiling of $\mathcal{R}$ with closed rectangular cells whose edges are parallel to the $X$ and $Y$ axes, and whose interiors have a pairwise empty intersection. The position of each cell $R$ is given by its center point $\left(x_{R}, y_{R}\right) \in \mathbb{R}^{2}$ and its length along $X$ and $Y$ axes by $\left(l_{R}^{x}, l_{R}^{y}\right) \in \mathbb{R}_{+}^{* 2}$.

This model permits to generalize many irregular image representations such as quadtrees, $k$ d-trees, run-length encodings, and the geometry of frames encoded with video coding standards like MPEG, H.264, etc.

We also define some topological objects on $\mathbb{I}$-grids, thanks to the following definitions.

Definition 3 (ve-adjacency and $e$-adjacency). Let $R_{1}$ and $R_{2}$ be two cells. $R_{1}$ and $R_{2}$ are ve-adjacent (vertex and edge adjacent) if :

$$
\text { or }\left\{\begin{array}{l}
\left|x_{R_{1}}-x_{R_{2}}\right|=\frac{l_{R_{1}}^{x}+l_{R_{2}}^{x}}{2} \text { and }\left|y_{R_{1}}-y_{R_{2}}\right| \leq \frac{l_{R_{1}}^{y}+l_{R_{2}}^{y}}{2} \\
\left|y_{R_{1}}-y_{R_{2}}\right|=\frac{l_{R_{1}}^{y}+l_{R_{2}}^{y}}{2} \text { and }\left|x_{R_{1}}-x_{R_{2}}\right| \leq \frac{l_{R_{1}}^{x}+l_{R_{2}}^{x}}{2}
\end{array}\right.
$$

$R_{1}$ and $R_{2}$ are e-adjacent (edge adjacent) if we consider an exclusive "or" and strict inequalities in the above ve-adjacency definition. The letter $k$ may be interpreted as e or ve in the following definitions.

An other important notion for our work is the concept of order on II-grids. In the following, we will consider the total order relations based on the cell borders. We denote the left, right, top and bottom borders of a cell $R$ respectively $R^{L}$, $R^{R}, R^{T}$ and $R^{B}$. The abscissa of $R^{L}$, for example, is equal to $x_{R}-\left(l_{R}^{x} / 2\right)$. In the following, we also denote by $\frac{\leq}{x}$ (resp. $\underset{y}{\leq}$ ) the natural order relation along $X$ (resp. $Y$ ) axis. It is legitimate to use the order $\underset{x}{\leq}$ on left and right borders of cells and the order $\underset{y}{\leq}$ on top and bottom borders of cells. 
Definition 4 (Order relations on an $\mathbb{I}$-grid). Let $R_{1}$ and $R_{2}$ be two cells of an $\mathbb{I}$-grid $G$. We define the total order relations $\preceq^{L}$ and $\preceq^{T}$, based on the cells borders:

$$
\begin{aligned}
& R_{1} \preceq^{L} R_{2} \Leftrightarrow R_{1}^{L}<_{x} R_{2}^{L} \vee\left(R_{1}^{L} \underset{x}{\bar{x}} R_{2}^{L} \wedge R_{1}^{T} \underset{y}{R_{2}^{T}}\right) ; \\
& R_{1} \preceq^{T} R_{2} \Leftrightarrow R_{1}^{T} \underset{y}{<} R_{2}^{T} \vee\left(R_{1}^{T} \underset{y}{=} R_{2}^{T} \wedge R_{1}^{L} \underset{x}{\leq} R_{2}^{L}\right) .
\end{aligned}
$$

A $k$-path from $R$ to $R^{\prime}$ is a sequence of cells $\left(R_{i}\right)_{1 \leq i \leq n}$ with $R=R_{1}$ and $R^{\prime}=R_{n}$ such that for any $i, 2 \leq i<n, R_{i}$ is $k$-adjacent to $R_{i-1}$ and $R_{i+1}$.

Definition 5 ( $k$-arc). Let $A=\left(R_{i}\right)_{1 \leq i \leq n}$ be a $k$-path from $R_{1}$ to $R_{n}$. Then $A$ is a $k$-arc iff each cell $R_{i}$ has exactly two $k$-adjacent cells in $A$ except $R_{1}$ and $R_{n}$ which have only one $k$-adjacent cell in $A$. The cells $R_{1}$ and $R_{n}$ are called the extremities of $A$.

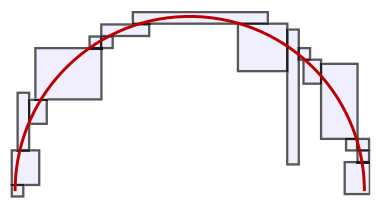

Fig. 2. In an $e$-arc on $\mathbb{I}$-grids, a $D I P$-circle may be recognized by resolving a separation problem between the extremities of each interface between two $e$-adjacent cells

Our purpose is now to extend the DIP-circle model we have previously presented to this kind of grids. The extension we use is natural.

Property 4 (Characterization of e-arcs of DIP-circles). Let $R_{1}$ and $R_{2}$ be two $e$-adjacent cells of $\mathrm{C}_{\mathrm{DIP}}(\mathbf{c}, r)$ such that they form a $e$-arc with their $e$-neighbors in $\mathrm{C}_{\mathrm{DIP}}(\mathbf{c}, r)$. Then, we have:

- both $R_{1}$ and $R_{2}$ deprived of their interface intersect the circle $\mathcal{C}(\mathbf{c}, r)$,

- the circle $\mathcal{C}(\mathbf{c}, r)$ intersects only in one point the interface between $R_{1}$ and $R_{1}$ deprived of its end points at a distance to $\mathbf{c}$ lower than $r$.

The reduction of the arc recognition problem in an $\mathbb{I}$-grid to a circle separation problem follows the same principle as in a regular grid: the parameters of the recognized $D I P$-circle are the same of those of a circle which separates the both extremities of each interface between two $e$-adjacent cells of the initial $e$-arc, as in Figure 2 .

\section{Application to Noisy Contour Reconstruction}

In this section, we attempt to build a global system of recognition of arcs of circles through digital contours. Our goal is to confront the concepts previously 
introduced to practical cases. At this point, it is mainly used to recognize circles, since it applies only to contours of disk-shaped objects. The limitation comes from the difficulties to deduce global $k$-arcs from any irregular isothetic object. At the end of the present paper, we point out some perspectives on how to overcome this limitation.

\subsection{Method}

The flowchart of our method is given in Figure 3 . Our arc reconstruction method is divided into three main steps: a multi-scale noise detection, a topological reconstruction of the resulting object and a translation into a circle separation problem.

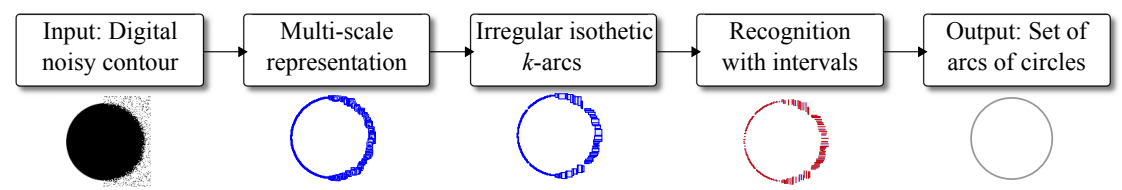

Fig. 3. The global scheme of our method, where an example leads to the recognition of one arc of circle (the complete circle)

The noise detector developed in [6] exploits the known asymptotic properties of maximal straight segments for flat parts, or smooth curved parts, of a digital contour. Asymptotic properties hold for finer and finer scale, but it appears that in practice, it fits also well for coarser and coarser scales. The contour is then subsampled at different scales and the asymptotic properties are tested. If they are not satisfied, the finer scales are removed and the properties tested again. Each contour point is then covered by a square cell, called meaningful box, whose size is the finest resolution determined by this scale detection. Finally, the higher the local amount of noise is, the bigger the meaningful box is. In Figure 4, we present the result of this process on a sample noisy image, the set $\mathcal{M}$ of the meaningful boxes deduced from the contour of the black object.

As shown in Figure 4(b), the meaningful boxes of $\mathcal{M}$ overlap and thus cannot be viewed as an irregular isothetic object directly (Definition 2). However each one contains a given number of pixels (at the initial resolution) so that the set of boxes covers a subset of the input image. This subset $\mathcal{P}$, which is an irregular isothetic object, is transformed into four $k$-arcs (Definition 5). Instead of using a unique order relation over the complete object $\mathcal{M}$, we use either $\preceq^{L}$ or $\preceq^{T}$, depending on the position of the treated cells. More precisely, we use the following procedure (see also Figure [5):

(1) We compute the barycenter $\overline{\mathbf{p}}$ of the input digital object, based on the center of the pixels belonging to $\mathcal{P}$.

(2) We construct two straight lines $\ell_{1}$ and $\ell_{2}$ passing through $\overline{\mathbf{p}}$, with slopes 1 and -1 . These lines define four quadrants in the Euclidean plane. 


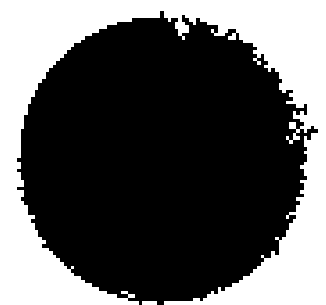

(a)

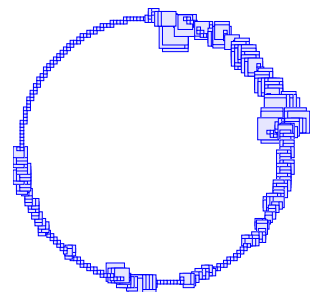

(b)

Fig. 4. We analyze the noise of a digital contour to get a multi-scale structure

(3) We use the topological reconstruction algorithm from [17] with $\preceq^{L}$ order for pixels of $\mathcal{P}$ in quadrants 1 and 3 and $\preceq^{T}$ order for pixels of $\mathcal{P}$ in quadrants 2 and 4.

The result is a set of four $e$-arcs, one in each quadrant defined by $\ell_{1}$ and $\ell_{2}$. Cells on both side of the boundary between two quadrant can overlap. For clarity issues, overlapping cells are not drawn in Figure 5.

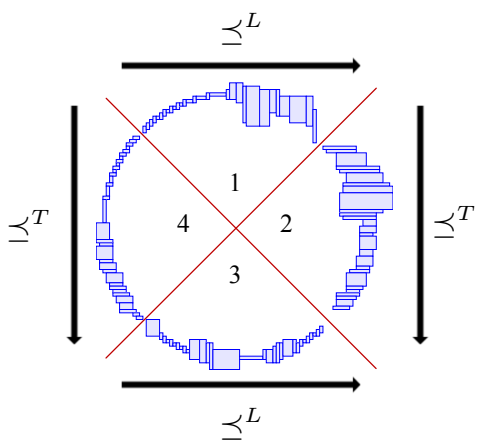

Fig. 5. The construction of the barycenter at the intersection of $\ell_{1}$ and $\ell_{2}$

Then, we consider the interface (Euclidean segments shared) between two consecutive cells in the $e$-arcs over the quadrants 1 to 4 , in the clockwise order. Finding circles inside the original noisy contour can now be considered as a problem of separation of the points defined as the extremities of these segments. To do so, we apply Algorithm 1 introduced by Roussillon et al. [16. It is online and its time complexity is basically $\mathcal{O}\left(n_{S}^{2}\right)\left(n_{S}\right.$ is the number of input segments), but may be optimized to $\mathcal{O}\left(n_{S}{ }^{4 / 3}\right)$ and even seems to be linear in practice.

Algorithm 1 first aims to recognize a set of straight lines $\mathcal{P}_{l}\left(A_{j}\right)$ passing through the input intervals (also known as preimage), from line 3 to 7 (Figure 6(a)). This recognition process is realized in an incremental way, by inserting input segments in the arc $A_{j}$. The end of this phase means that no straight line can pass through $A_{j}$. Then, a fixed point $\mathbf{p}$ is selected from one of the extremities of the interval that fails the first step. The $\operatorname{arc} A_{j}$ is updated so that it represents 


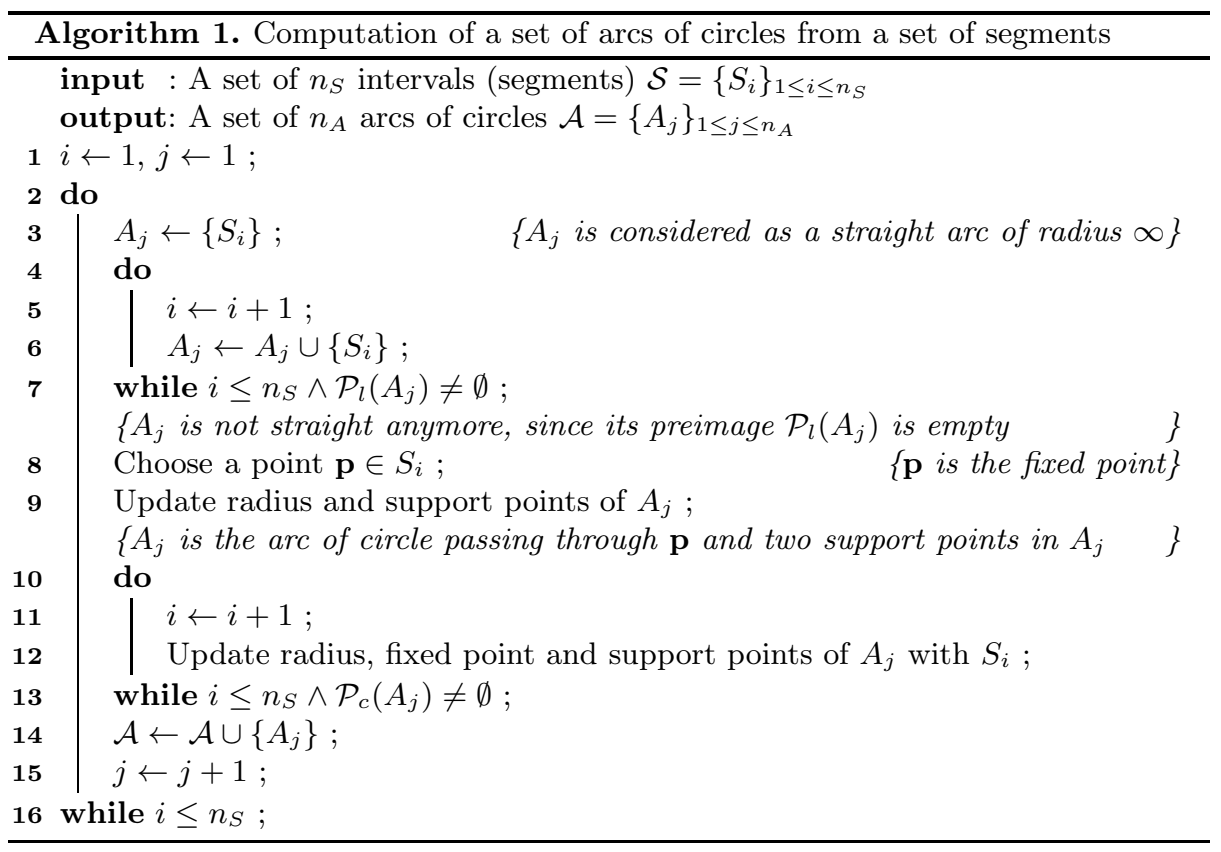

a circle passing through $\mathbf{p}$ and two support points of $A_{j}$. These points belong to the partial upper and lower convex envelopes of the set of points to separate [16]. While intervals are added incrementally, the support points and the fixed point are updated to fit the circle at best to the input segments (Figure 6(b)). This second phase, from line 10 to 13 in Algorithm 1, finishes once the set of circles passing through $A_{j}$, denoted by $\mathcal{P}_{c}\left(A_{j}\right)$, is empty. The arc $A_{j}$ is added to the set of recognized arcs $\mathcal{A}$, lines 14 and 15 , and a new arc may be constructed. If the number of segments $n_{S}$ is achieved at this moment, the result only contains one arc, as in Figure 6(c).

\subsection{Experiments}

Synthetic Image. Throughout Section 4.1, we illustrate the different steps of our method with the example of a circle with non uniform noise distribution. It is reconstructed as a single circle with parameters different from those of the circle used to generate the image (see the result in Figure 66(c)). The circle separation problem our method solves has generally many solutions and Algorithm 1 chooses only one. We have checked that the original circle is also one of the solutions.

Sun Image at Different Resolutions. In Figure 7, we consider a sun image of size $n \times n$, with two different resolutions $n=512$ and $n=1024$. A contour of the sun is extracted by a simple thresholding. In both cases, our method succeeds in reconstructing only a single circle. 

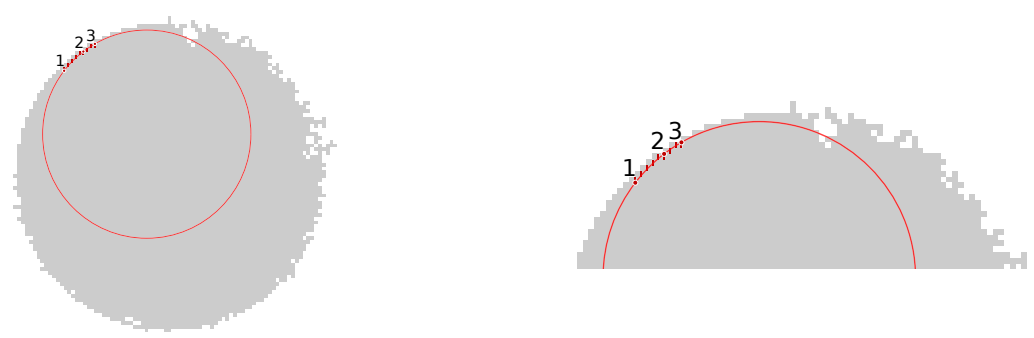

(a)
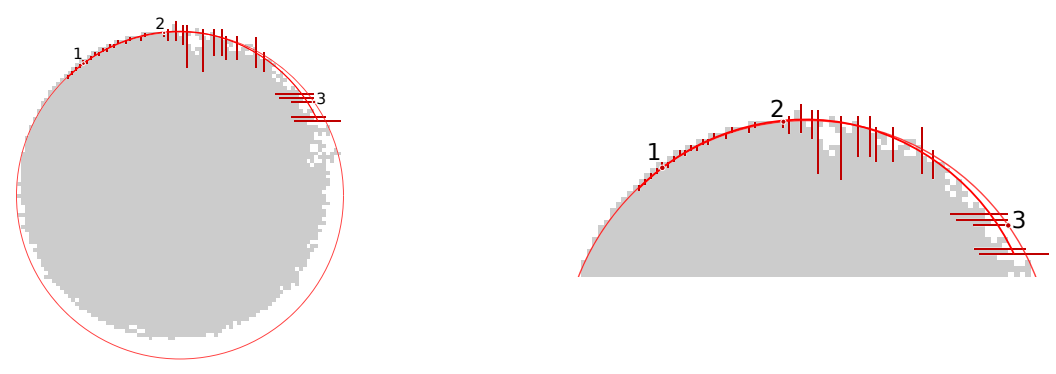

(b)

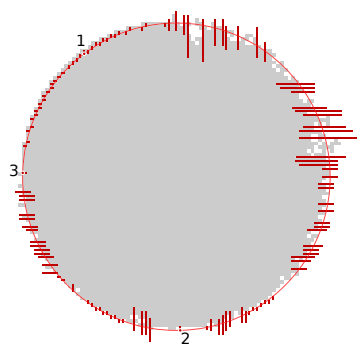

(c)

Fig. 6. The first step of Algorithm 1 consists in recognizing a segment (a). When this phase stops, a first arc of circle is constructed, passing through a fixed point (indexed 3 ), and two support points (indexed 1 and 2). This process continues, and these three points are updated, leading to the update of the circle recognized (b). The process stops when all the input intervals are treated (c). 
When we consider the highest resolution available for this picture $(n=2400)$, we obtain a decomposition of 33 arcs of circles. At this scale the small details of the digital contour are no more considered as noise and the algorithm is now able to detect arcs defined in finer resolution.

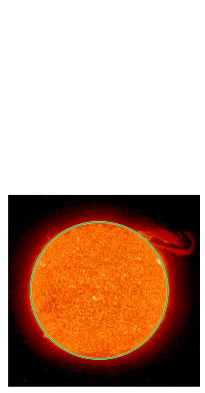

(a) $n=512$

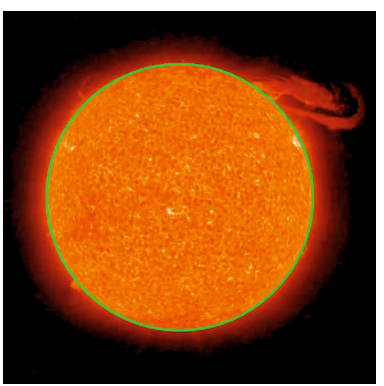

(b) $n=1024$

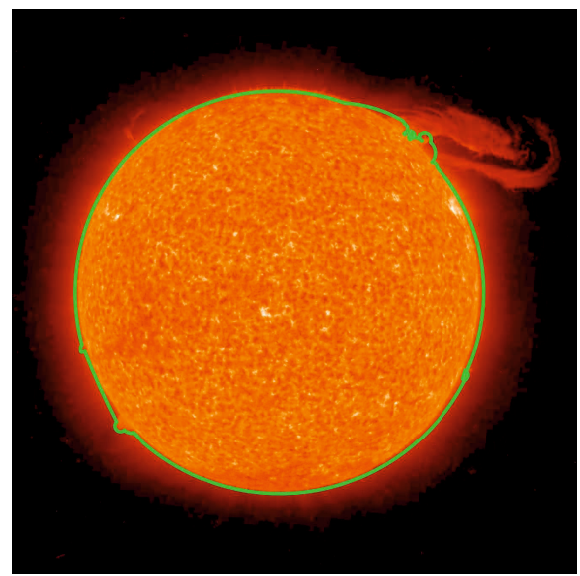

(c) $n=2400$

Fig. 7. The extraction of one circle realized on an image of Sun, for the 512 and 1024 resolutions. For the original resolution (2400), our method leads to a decomposition of 33 arcs of circles.

Non-circular Shapes. We have also tested our method on an image of road sign (of $520 \times 340$ pixels), where the contour is roughly elliptic. In this case again, we are able to extract arcs of circles close to the real contour, as illustrated in Figure 8 .

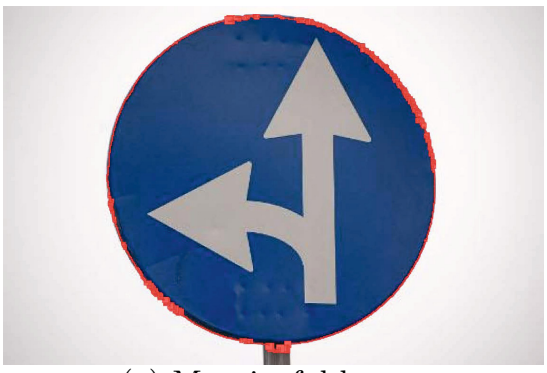

(a) Meaningful boxes

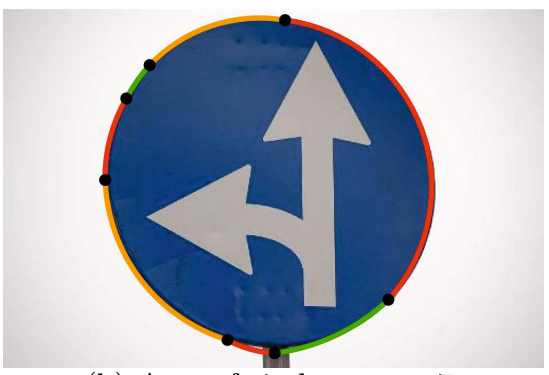

(b) Arcs of circles, $n_{A}=7$

Fig. 8. Extraction of the meaning boxes from the road sign (a), and its associated decomposition into 7 arcs of circles (b) 


\section{Conclusion and Future Works}

In the present paper, we have introduced a new circle model close to the Kovalevsky's one. Thanks to its links with the inter-pixel boundary, it is provided with interesting properties to be recognized through the resolution of a circle separation problem. Moreover, it extends nicely to irregular isothetic grids and objects.

From a practical point of view, we have designed a simple arc recognition scheme. It involves recent results in the fields of digital geometry about noise detection, isothetic irregular objects and digital circles. Each step can be proceeded in linear time with respect to the length of the digital contour analyzed. Applications to synthetic images ensure that we are able to evaluate a promising method, whereas applications to real images show its concrete interest and efficiency. Nevertheless, deeper tests and comparisons with existing recognition schemes should be performed.

In future works, we plan to improve the decomposition of the irregular isothetic object into $e$-arcs. In the scheme presented in the present paper, we can only process disk-shaped objects. This is due to the naive decomposition of the isothetic object used to reconstruct $e$-arcs : we break it, according to the case of circles, into quadrants where the total order relation $\preceq^{L}$ applies and quadrants where the total order relation $\preceq^{T}$ applies. This limitation can be overcome by adapting the decomposition to the treated object. A first approach could consist in recognizing segments in the set of the meaning boxes; their directions are indeed sufficient to choose the appropriate order relation for each part of the contour. A second approach could consist in computing the isothetic objects associated to each total order relation. Then, an approriate decomposition is a cover of the set of meaningful boxes by $e$-arcs of these isothetic objects.

The decomposition in both arcs and segments of noisy contours is also a question we intend to address. Indeed, tools and approaches for both primitives are similar 1819 .

\section{References}

1. Coeurjolly, D., Gerard, Y., Reveillès, J.P., Tougne, L.: An elementary algorithm for digital arc segmentation. Dis. Applied Maths 139(1-3), 31-50 (2004)

2. Coeurjolly, D., Vacavant, A.: Separable Distance Transformation and its Applications. In: Brimkov, V., Barneva, R. (eds.) Digital Geometry Algorithms. Theoretical Foundations and Applications to Computational Imaging. Springer (2012)

3. Damaschke, P.: The linear time recognition of digital arcs. Pat. Rec. Letters 16(5), 543-548 (1995)

4. Debled-Rennesson, I., Feschet, F., Rouyer-Degli, J.: Optimal blurred segments decomposition of noisy shapes in linear time. Computers \& Graphics 30(1), 30-36 (2006)

5. Duda, R.O., Hart, P.E.: Use of the Hough transformation to detect lines and curves in pictures. Com. of ACM 15(1), 11-15 (1972) 
6. Kerautret, B., Lachaud, J.-O.: Multi-scale Analysis of Discrete Contours for Unsupervised Noise Detection. In: Wiederhold, P., Barneva, R.P. (eds.) IWCIA 2009. LNCS, vol. 5852, pp. 187-200. Springer, Heidelberg (2009)

7. Kerautret, B., Lachaud, J.: Meaningful scales detection along digital contours for unsupervised local noise estimation. IEEE Pat. Analysis and Machine Intel. 34(12), 2379-2392 (2012)

8. Kerautret, B., Lachaud, J.-O., Nguyen, T.P.: Circular Arc Reconstruction of Digital Contours with Chosen Hausdorff Error. In: Debled-Rennesson, I., Domenjoud, E., Kerautret, B., Even, P. (eds.) DGCI 2011. LNCS, vol. 6607, pp. 247-259. Springer, Heidelberg (2011)

9. Kovalevsky, V.A.: New definition and fast recognition of digital straight segments and arcs. In: ICPR 1990, vol. 2, pp. 31-34 (1990)

10. Lamiroy, B., Fritz, L., Gaucher, O.: Robust Circle Detection. In: ICDAR 2007, pp. 526-530 (2007)

11. Megiddo, N.: Linear programming in linear time when the dimension is fixed. Jour. of ACM 31(1), 114-127 (1984)

12. Nguyen, T.P., Debled-Rennesson, I.: Arc Segmentation in Linear Time. In: Real, P., Diaz-Pernil, D., Molina-Abril, H., Berciano, A., Kropatsch, W. (eds.) CAIP 2011, Part I. LNCS, vol. 6854, pp. 84-92. Springer, Heidelberg (2011)

13. Nguyen, T.P., Kerautret, B., Debled-Rennesson, I., Lachaud, J.-O.: Unsupervised, Fast and Precise Recognition of Digital Arcs in Noisy Images. In: Bolc, L., Tadeusiewicz, R., Chmielewski, L.J., Wojciechowski, K. (eds.) ICCVG 2010, Part I. LNCS, vol. 6374, pp. 59-68. Springer, Heidelberg (2010)

14. Pham, S.: Digital circles with non-lattice point centers. The Visual Computer 9, 1-24 (1992)

15. Ayala-Ramírez, V., Garcia-Capulin, C.H., Pérez-García, A., Sánchez-Yáñez, R.E.: Circle detection on images using genetic algorithms. Pat. Rec. Letters 27(6), 652657 (2006)

16. Roussillon, T., Tougne, L., Sivignon, I.: On Three Constrained Versions of the Digital Circular Arc Recognition Problem. In: Brlek, S., Reutenauer, C., Provençal, X. (eds.) DGCI 2009. LNCS, vol. 5810, pp. 34-45. Springer, Heidelberg (2009)

17. Vacavant, A., Coeurjolly, D., Tougne, L.: Topological and Geometrical Reconstruction of Complex Objects on Irregular Isothetic Grids. In: Kuba, A., Nyúl, L.G., Palágyi, K. (eds.) DGCI 2006. LNCS, vol. 4245, pp. 470-481. Springer, Heidelberg (2006)

18. Vacavant, A., Roussillon, T., Kerautret, B.: Unsupervised Polygonal Reconstruction of Noisy Contours by a Discrete Irregular Approach. In: Aggarwal, J.K., Barneva, R.P., Brimkov, V.E., Koroutchev, K.N., Korutcheva, E.R. (eds.) IWCIA 2011. LNCS, vol. 6636, pp. 398-409. Springer, Heidelberg (2011)

19. Vacavant, A., Roussillon, T., Kerautret, B., Lachaud, J.-O.: A Combined MultiScale/Irregular Algorithm for the Vectorization of Noisy Digital Contours. Computer Vision and Image Understanding (to appear, 2013) 